\title{
Investigation on Stability Properties of Hierarchical Co-Simulation
}

\author{
Irene Hafner ${ }^{1 *}$, Niki Popper ${ }^{2}$ \\ ${ }^{1}$ dwh GmbH, Neustiftgasse 57-59, 1070 Vienna, Austria; *irene.hafner@dwh.at \\ ${ }^{2}$ Institute of Information Systems Engineering, Research Unit of Information and Software Engineering, TU Wien, \\ Favoritenstraße 9-11, 1040 Vienna, Austria
}

SNE 31(1), 2021, 17-24, DOI: 10.11128/sne.31.tn.10553

Received: November 10, 2020 (Selected ASIM vSST 2020

Post-conf. Publ.); Revised January 10, 2021;

Accepted: February 2, 2021

SNE - Simulation Notes Europe, ARGESIM Publisher Vienna,

ISSN Print 2305-9974, Online 2306-0271, www.sne-journal.org

Abstract. This paper introduces the concept of hierarchical co-simulation and presents an investigation on stability properties of this method. In conventional cosimulation methods, all participating simulations are executed on the same level via one co-simulation. Hierarchical co-simulation, on the other hand, enables the introduction of several levels of co-simulation by allowing participating subsystems to consist of co-simulated systems themselves, thus nesting co-simulations within cosimulations. While on the one hand, certain stability issues can arise by the introduction of more co-simulation layers, this method enables the usage of different synchronization references for parts of the overall system according to varying dependencies between the subsystems, which can increase accuracy and numerical stability.

\section{Introduction}

Co-simulation has become an important instrument to approach the simulation of large-scale heterogeneous systems in recent years. While definitions for the term co-simulation vary depending on the field of origin, in this paper we refer (in accordance with the terminology found in [1]) to co-simulation as the coupling of two or more simulations which differ in at least one aspect out of simulation tool, solver algorithm or step size.

Hierarchical approaches or multi-level descriptions have already been introduced in other fields within modeling and simulation (f.i. DEVS [2], multi-level agent-based modeling [3], MPC [4] or partitioned multi-rate approaches [5]). However, hierarchical cosimulation as explained in the following has to the authors' knowledge not been investigated up to now, although several frameworks and standards do not prohibit the realization of further co-simulations within a co-simulation. The idea is illustrated in Figure 1.

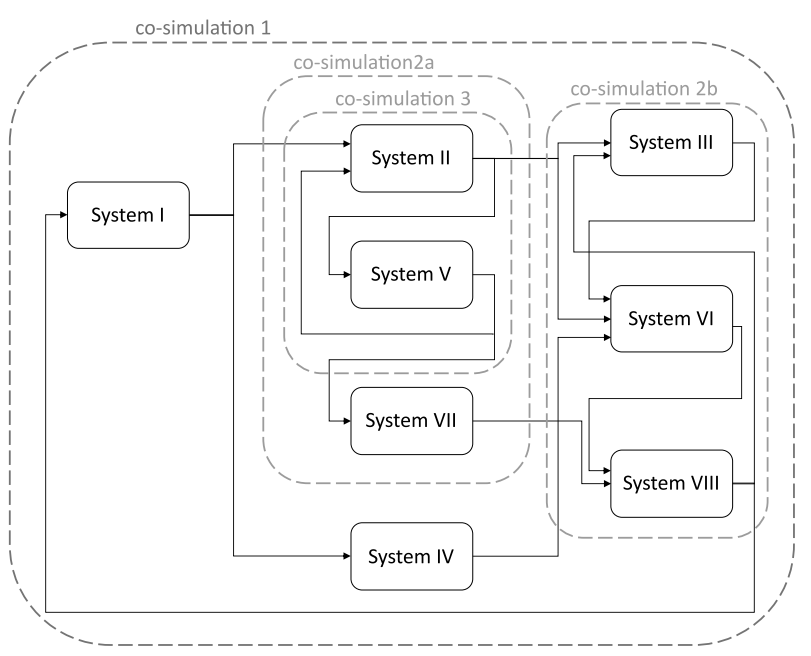

Figure 1: Schematic depiction of a hierarchical co-simulation approach.

In a traditional co-simulation approach all eight participating subsystems would have been co-simulated in one overall co-simulation, probably requiring all systems to synchronize at the same points in time. In the hierarchical approach, systems III, VI and VIII are coupled in another co-simulation (2b) as well as systems II and $\mathrm{V}$, the coupled system of which (co-simulation 3 ) is again co-simulated with system VII, before the resulting co-simulation (2a) represents a system coupled in the top-level co-simulation 1.

A coupling structure like this could be motivated by the usage of different synchronization intervals on every 
co-simulation level, thus enabling frequent exchanges between subsystems which are sensitive to changes in their respective exchanged values while allowing larger communication intervals with other, slower reacting system parts, which can speed up the overall execution. In the course of this paper, we will show that these ideas are valid and that a hierarchically structured cosimulation approach indeed allows to enhance stability at a low computational cost.

\section{Consistency}

A valid method to bound the global co-simulation error is local error control, which justifies to investigate the consistency error, i.e. the error of the method in one step. For traditional co-simulation, it has been shown that consistency can be maintained, but possibly reduced to the extrapolation order of values from other systems, see for example $[6,17]$. Since consistency is defined locally (i.e. per step), and it is a property regarded for the limit of step size $h \rightarrow 0$, the value present at the most recent point in time where the method sets a step is considered to be the exact solution - a property that is not affected by the method used in the respective other subsystems or the time steps and further synchronizations happening there in-between. This means that consistency in hierarchical co-simulation is also maintained with its order depending on the applied extrapolation method.

\section{Zero-stability}

Zero-stability, i.e. convergence of a method for infinitesimal step sizes, has been analyzed for certain cosimulation approaches in [7], on which we base our investigation. The mathematical description of coupled DAEs is given in [7] as follows:

$$
\begin{aligned}
\dot{\boldsymbol{x}}^{i}(t) & =\boldsymbol{f}^{i}\left(\boldsymbol{x}^{i}, \boldsymbol{u}^{i}, t\right), \quad \boldsymbol{x}^{i}\left(t_{0}\right)=\boldsymbol{x}_{0}^{i} \\
\boldsymbol{y}^{i}(t) & =\boldsymbol{g}^{i}\left(\boldsymbol{x}^{i}, \boldsymbol{u}^{i}, t\right)
\end{aligned}
$$

with $i=I, \ldots, N, \boldsymbol{x}^{i} \in \mathbb{R}^{n_{x}^{i}}, \boldsymbol{u}^{i} \in \mathbb{R}^{n_{u}^{i}}, \boldsymbol{y}^{i} \in \mathbb{R}^{n_{y}^{i}}$ and

$$
\boldsymbol{u}^{i}=\boldsymbol{L}^{i} \boldsymbol{y}
$$

where

$$
\boldsymbol{L}^{i}=\left[\begin{array}{lllllll}
\boldsymbol{L}^{i, I} & \ldots & \boldsymbol{L}^{i, i-1} & 0 & \boldsymbol{L}^{i, i+1} & \ldots & \boldsymbol{L}^{i, N}
\end{array}\right],
$$

$$
\boldsymbol{y}=\left[\begin{array}{lllllll}
\boldsymbol{y}^{I} & \ldots & \boldsymbol{y}^{i-1} & \boldsymbol{y}^{i} & \boldsymbol{y}^{i+1} & \ldots & \boldsymbol{y}^{N}
\end{array}\right]^{T}
$$

with $\boldsymbol{L}^{i, j} \in \mathbb{R}^{n_{u}^{i} \times n_{y}^{j}} \quad \forall i, j \in\{I, \ldots, N\}$ and the elements of $\boldsymbol{L}^{i, j}$ being equal to zero or one.

Under certain assumptions (given in [7], p. 100), the outputs can be written as $\boldsymbol{y}^{i}=\overline{\boldsymbol{g}}^{i}\left(\boldsymbol{x}^{i}\right)+\boldsymbol{D}^{i}\left(\boldsymbol{x}^{i}\right) \boldsymbol{u}^{i}$, yielding the discretized output equations

$$
\boldsymbol{y}_{k+1}^{i}=\overline{\boldsymbol{g}}^{i}+\boldsymbol{D}^{i} \boldsymbol{u}_{k}^{i}
$$

with constant $\overline{\boldsymbol{g}}^{i}, \boldsymbol{D}^{i}$. Using this, it holds for the outputs of global system

$$
\boldsymbol{y}_{k+1}=\overline{\boldsymbol{g}}+\underbrace{\left[\begin{array}{cccc}
\mathbf{0} & \boldsymbol{D}^{I} \boldsymbol{L}^{I, I I} & \ldots & \boldsymbol{D}^{I} \boldsymbol{L}^{I, N} \\
\boldsymbol{D}^{I I} \boldsymbol{L}^{I I, I} & \mathbf{0} & \ldots & \boldsymbol{D}^{I I} \boldsymbol{L}^{I I, N} \\
\vdots & \vdots & \ddots & \vdots \\
\boldsymbol{D}^{N} \boldsymbol{L}^{N, I} & \boldsymbol{D}^{N} \boldsymbol{L}^{N, I I} & \ldots & \mathbf{0}
\end{array}\right]}_{=: D} \boldsymbol{y}_{k}
$$

that stability is guaranteed if the spectral radius $\rho$ of $\boldsymbol{D}$ is less than or equal to 1 . This is fulfilled in particular if $\rho(\boldsymbol{D})=0$ which for the case of two participating subsystems means that there is no algebraic loop.

To determine zero-stability properties of hierarchical co-simulation, a co-simulation of $N$ systems is considered, where w.l.o.g. systems $M$ to $N$ are combined in a second-level co-simulation as depicted in Figure 2.

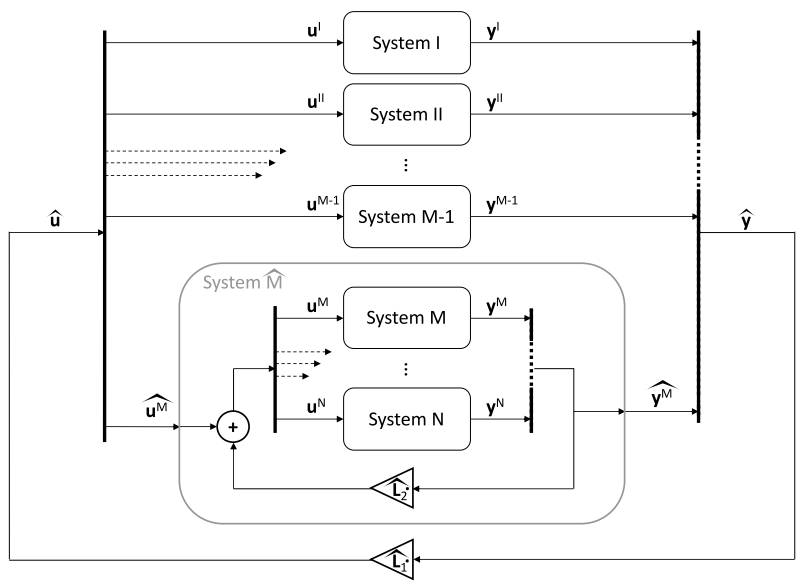

Figure 2: Hierarchical co-simulation of $N$ systems on two levels.

System $\widehat{M}$ replaces systems $M$ to $N$ of the original co-simulation on one level (coupled via 1c and called $C S_{0}$ henceforth). We obtain coupling equations (4) for 
the upper co-simulation level $C S_{1}$ :

$$
\left[\begin{array}{c}
\boldsymbol{u}^{I} \\
\boldsymbol{u}^{I I} \\
\vdots \\
\boldsymbol{u}^{M-1} \\
\widehat{\boldsymbol{u}^{M}}
\end{array}\right]=\widehat{\boldsymbol{L}_{1}}\left[\begin{array}{c}
\boldsymbol{y}^{I} \\
\boldsymbol{y}^{I I} \\
\vdots \\
\boldsymbol{y}^{M-1} \\
\widehat{\boldsymbol{y}^{M}}
\end{array}\right]
$$

with

$$
\widehat{\boldsymbol{L}_{1}}=\left[\begin{array}{ccccc}
\mathbf{0} & \boldsymbol{L}^{I, I I} & \ldots & \boldsymbol{L}^{I, M-1} & \widehat{\boldsymbol{L}^{I, M}} \\
\boldsymbol{L}^{I I, I} & \mathbf{0} & \ldots & \boldsymbol{L}^{I I, M-1} & \widehat{\boldsymbol{L}^{I I, M}} \\
\vdots & \vdots & \ddots & \vdots & \vdots \\
\boldsymbol{L}^{M-1, I} & \boldsymbol{L}^{M-1, I I} & \ldots & \mathbf{0} & \widehat{\boldsymbol{L}^{M-1, M}} \\
\widehat{\boldsymbol{L}^{M, I}} & \widehat{\boldsymbol{L}^{M, I I}} & \ldots & \widehat{\boldsymbol{L}^{M, M-1}} & \mathbf{0}
\end{array}\right]
$$

and $\widehat{\boldsymbol{u}^{M}}$ as input to the new subsystem $\widehat{M}, \widehat{\boldsymbol{y}^{M}}$ as its output and

$$
\begin{aligned}
& \widehat{\boldsymbol{L}^{i, M}}=\left[\begin{array}{llll}
\boldsymbol{L}^{i, M} & \boldsymbol{L}^{i, M+1} & \ldots & \boldsymbol{L}^{i, N}
\end{array}\right], i=I \ldots M-1 \\
& \widehat{\boldsymbol{L}^{M, i}} \\
& =\left[\begin{array}{c}
\boldsymbol{L}^{M, i} \\
\boldsymbol{L}^{M+1, i} \\
\vdots \\
\boldsymbol{L}^{N, i}
\end{array}\right], i=I \ldots M-1 .
\end{aligned}
$$

Thus, the only difference between $\boldsymbol{L}$ and $\widehat{\boldsymbol{L}_{1}}$ is the increased number of zeroes in the lower right corner. The discretized output equations of $C S_{1}$ are (5):

$$
\begin{array}{cc}
\boldsymbol{y}_{k+1}^{I} & =\overline{\boldsymbol{g}}^{I}+\boldsymbol{D}^{I} \boldsymbol{u}_{k}^{I} \\
\boldsymbol{y}_{k+1}^{I I} & =\overline{\boldsymbol{g}}^{I I}+\boldsymbol{D}^{I I} \boldsymbol{u}_{k}^{I I} \\
& \vdots \\
\widehat{\boldsymbol{y}_{k+1}^{M}} & =\widehat{\boldsymbol{g}^{M}}+\widehat{\boldsymbol{D}^{M}} \widehat{\boldsymbol{u}_{k}^{M}}
\end{array}
$$

While $\widehat{\boldsymbol{y}^{M}}$ in general corresponds to the stacked output vectors $\boldsymbol{y}^{M} \ldots \boldsymbol{y}^{N}$ of $C S_{0}$, the input vectors don't as the coupling with the outputs of systems $M$ to $N$ is considered within the new system $\widehat{M}$, cf. Figure 2 and (6).

The outputs of the global system can with (5) be written as

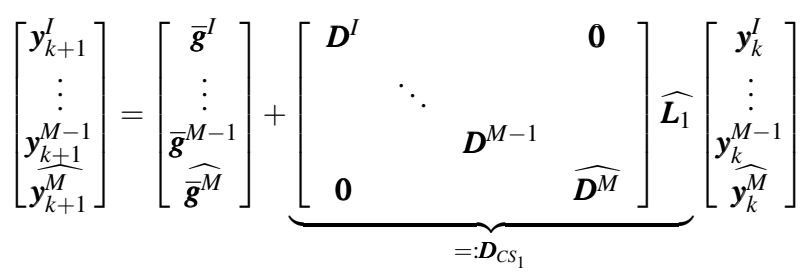

In analogy to the case of one co-simulation level, the cosimulation of the upper level is stable if $\rho\left(D_{C S_{1}}\right) \leq 1$. The only unknown in comparison to $\boldsymbol{D}$ of $C S_{0}$ is $\widehat{\boldsymbol{D}^{M}}$, for which we have to take a look at the second-level cosimulation $\mathrm{CS}_{2}$. The coupling equations within this system can be written (cf. Figure 2) as follows:

$$
\left[\begin{array}{c}
\boldsymbol{u}_{k}^{M} \\
\boldsymbol{u}_{k}^{M+1} \\
\vdots \\
\boldsymbol{u}_{k}^{N-1} \\
\boldsymbol{u}_{k}^{N}
\end{array}\right]=\widehat{\boldsymbol{L}_{2}} \cdot\left[\begin{array}{c}
\boldsymbol{y}_{k}^{M} \\
\boldsymbol{y}_{k}^{M+1} \\
\vdots \\
\boldsymbol{y}_{k}^{N-1} \\
\boldsymbol{y}_{k}^{N}
\end{array}\right]+\widehat{\boldsymbol{u}_{k}^{M}}
$$

where

$\widehat{\boldsymbol{L}_{\mathbf{2}}}=\left[\begin{array}{ccccc}\mathbf{0} & \boldsymbol{L}^{M, M+1} & \ldots & \boldsymbol{L}^{M, N-1} & \boldsymbol{L}^{M, N} \\ \boldsymbol{L}^{M+1, M} & \mathbf{0} & \ldots & \boldsymbol{L}^{M+1, N-1} & \boldsymbol{L}^{M+1, N} \\ \vdots & \vdots & \ddots & \vdots & \vdots \\ \boldsymbol{L}^{N-1, M} & \boldsymbol{L}^{N-1, M+1} & \ldots & \mathbf{0} & \boldsymbol{L}^{N-1, N} \\ \boldsymbol{L}^{N, M} & \boldsymbol{L}^{N, M+1} & \ldots & \boldsymbol{L}^{N, N-1} & \mathbf{0}\end{array}\right]$

The discretized output equations are

$$
\boldsymbol{y}_{k+1}^{i}=\overline{\boldsymbol{g}}^{i}+\boldsymbol{D}^{i} \boldsymbol{u}_{k}^{i}, i=M \ldots N .
$$

Thus follows for the global output of $\mathrm{CS}_{2}$

$$
\widehat{\boldsymbol{y}_{k+1}^{M}}=\left[\begin{array}{c}
\boldsymbol{y}_{k+1}^{M} \\
\vdots \\
\boldsymbol{y}_{k+1}^{N}
\end{array}\right]=\widehat{\bar{g}^{M}}+\left[\begin{array}{ccc}
\boldsymbol{D}^{M} & \ldots & \mathbf{0} \\
\vdots & \ddots & \vdots \\
\mathbf{0} & \ldots & \boldsymbol{D}^{N}
\end{array}\right] \widehat{\boldsymbol{u}_{k}^{M}}
$$

with

$$
\widehat{\overline{\boldsymbol{g}}^{M}}=\left[\begin{array}{c}
\overline{\boldsymbol{g}}^{M} \\
\vdots \\
\overline{\boldsymbol{g}}^{N}
\end{array}\right]+\left[\begin{array}{ccc}
\boldsymbol{D}^{M} & \ldots & \mathbf{0} \\
\vdots & \ddots & \vdots \\
\mathbf{0} & \ldots & \boldsymbol{D}^{N}
\end{array}\right] \widehat{\boldsymbol{L}_{2}}\left[\begin{array}{c}
\boldsymbol{y}_{k}^{M} \\
\vdots \\
\boldsymbol{y}_{k}^{N}
\end{array}\right]
$$

The part containing $\boldsymbol{y}_{k}^{i}, i=M \ldots N$ can be included in $\widehat{\overline{\boldsymbol{g}}^{M}}$ as these are only internal states of $C S_{2}$ which are unknown in $C S_{1}$. Hence we obtain

$$
\widehat{\boldsymbol{D}^{M}}=\left[\begin{array}{ccc}
\boldsymbol{D}^{M} & \ldots & \mathbf{0} \\
\vdots & \ddots & \vdots \\
\mathbf{0} & \ldots & \boldsymbol{D}^{N}
\end{array}\right]
$$


which yields

$$
\boldsymbol{D}_{C S_{1}}=\left[\begin{array}{cccc}
\mathbf{0} & \boldsymbol{D}^{I} \boldsymbol{L}^{I, I I} & \cdots & \boldsymbol{D}^{I} \widehat{\boldsymbol{L}^{I, M}} \\
\boldsymbol{D}^{I I} \boldsymbol{L}^{I I, I} & \mathbf{0} & \cdots & \boldsymbol{D}^{I I} \boldsymbol{L}^{I I, M} \\
\vdots & \ddots & \ldots & \vdots \\
\boldsymbol{D}^{M-1} \boldsymbol{L}^{M-1, I} & \ldots & \widehat{0} & \boldsymbol{D}^{M-1} \widehat{\boldsymbol{L}^{M-1, M}} \\
\widehat{\boldsymbol{D}^{M}} \boldsymbol{L}^{M, I} & \cdots & \widehat{\boldsymbol{D}^{M}} \boldsymbol{L}^{\widehat{M, M-1}} & \mathbf{0}
\end{array}\right] .
$$

Due to

$$
\widehat{\boldsymbol{D}^{M}} \widehat{\boldsymbol{L}^{M, i}}=\left[\begin{array}{ccc}
\boldsymbol{D}^{M} & \ldots & \mathbf{0} \\
\vdots & \ddots & \vdots \\
\mathbf{0} & \ldots & \boldsymbol{D}^{N}
\end{array}\right] \cdot\left[\begin{array}{c}
\boldsymbol{L}^{M, i} \\
\boldsymbol{L}^{M+1, i} \\
\vdots \\
\boldsymbol{L}^{N, i}
\end{array}\right]=\left[\begin{array}{c}
\boldsymbol{D}^{M} \boldsymbol{L}^{M, i} \\
\boldsymbol{D}^{M} \boldsymbol{L}^{M+1, i} \\
\vdots \\
\boldsymbol{D}^{N} \boldsymbol{L}^{N, i}
\end{array}\right]
$$

and

$$
\begin{aligned}
\boldsymbol{D}^{i} \widehat{\boldsymbol{L}^{i, M}} & =\boldsymbol{D}^{i} \cdot\left[\begin{array}{llll}
\boldsymbol{L}^{i, M} & \boldsymbol{L}^{i, M+1} & \ldots & \boldsymbol{L}^{i, N}
\end{array}\right] \\
& =\left[\begin{array}{llll}
\boldsymbol{D}^{i} \boldsymbol{L}^{i, M} & \boldsymbol{D}^{i} \boldsymbol{L}^{i, M+1} & \ldots & \boldsymbol{D}^{i} \boldsymbol{L}^{i, N}
\end{array}\right]
\end{aligned}
$$

the only difference compared to matrix $\boldsymbol{D}$ of system $C S_{0}$ is the increased number of zeroes in the lower right corner. In the following, we try to use this to gain information on the properties of the spectral radius of $\boldsymbol{D}_{C S_{1}}$ using knowledge on $\rho(\boldsymbol{D})$.

We know that for every matrix norm $\|$.$\| and arbitrary$ matrix $\boldsymbol{A}=\left(a_{i j}\right) ; i=1, \ldots, m ; j=1, \ldots, n ; m, n \in \mathbb{N}$

$$
\rho(A) \leq\|\boldsymbol{A}\|
$$

holds ([8], Thm. 5.6.9).

If we consider $\|\cdot\|_{\infty}$ given as

$$
\|\boldsymbol{A}\|_{\infty}=\max _{i=1, \ldots, m} \sum_{j=1}^{n}\left|a_{i j}\right|
$$

we see at once that $\left\|\boldsymbol{D}_{C S_{1}}\right\|_{\infty} \leq\|\boldsymbol{D}\|_{\infty}$. Unfortunately, this does not imply $\rho\left(\boldsymbol{D}_{C S_{1}}\right) \leq \rho(\boldsymbol{D})$, see f.i. the following example: Let matrices $\boldsymbol{A}_{1}$ and $\boldsymbol{A}_{2}$ given as

$\boldsymbol{A}_{1}=\left[\begin{array}{cccc}0 & 0.1 & 0.5 & 0 \\ 0.1 & 0 & 0 & 0.5 \\ 0.2 & 0 & 0 & 0 \\ 0 & 0.2 & 0 & 0\end{array}\right], \quad \boldsymbol{A}_{2}=\left[\begin{array}{cccc}0 & 0.1 & 0.5 & 0 \\ 0.1 & 0 & 0 & 0.5 \\ 0.2 & 0 & 0 & -0.1 \\ 0 & 0.2 & -0.1 & 0\end{array}\right]$.

Here $\left\|\boldsymbol{A}_{1}\right\|_{\infty}=\left\|\boldsymbol{A}_{2}\right\|_{\infty}=0.6$ but

$\rho\left(\boldsymbol{A}_{1}\right) \approx 0.3702>\rho\left(\boldsymbol{A}_{2}\right) \approx 0.3317$. This means that in general, stability for hierarchical co-simulation has to be determined anew, even if the starting point is a zero-stable co-simulation on one level. An exception is the case where not only $\rho(\boldsymbol{D}) \leq 1$ but also $\|\boldsymbol{D}\|_{\infty} \leq 1$, as from this follows further

$$
\rho\left(\boldsymbol{D}_{C S_{1}}\right) \leq\left\|\boldsymbol{D}_{C S_{1}}\right\|_{\infty} \leq\|\boldsymbol{D}\|_{\infty} \leq 1
$$

which ensures zero-stability of the co-simulation on the upper level $C S_{1}$.

For the stability properties of the coupling in $C S_{2}$, we are interested in the input-output dependencies within the system only, thus we need to look at the spectral radius of $\boldsymbol{D}_{\mathrm{CS}_{2}}$, which results from (8):

$$
\boldsymbol{D}_{C S_{2}}=\left[\begin{array}{ccc}
\boldsymbol{D}^{M} & \ldots & \mathbf{0} \\
\vdots & \ddots & \vdots \\
\mathbf{0} & \ldots & \boldsymbol{D}^{N}
\end{array}\right] \widehat{\boldsymbol{L}_{2}}
$$

Since we see that $\boldsymbol{D}_{C S_{2}}$ is composed of a submatrix of $\boldsymbol{D}$, here again $\left\|\boldsymbol{D}_{C S_{2}}\right\|_{\infty} \leq\|\boldsymbol{D}\|_{\infty}$ holds, and thus $\rho\left(\boldsymbol{D}_{C S_{2}}\right)$ has to be determined separately only if $\|\boldsymbol{D}\|_{\infty}>1$.

To sum up, we can conclude that zero-stability of hierarchical co-simulation can be determined analogously to customary co-simulation on one level. To this end, the matrices referring to the global system outputs on every co-simulation level have to be examined - except for the cases where the origin is a stable co-simulation with matrix $\boldsymbol{D}$ fulfilling $\|\boldsymbol{D}\|_{\infty} \leq 1$, which is satisfied in particular for couplings where no feed-through occurs in at least one system, so $\|\boldsymbol{D}\|_{\infty}=\rho(\boldsymbol{D})=0$. These considerations, of course, can directly be taken further and applied to more than two levels of co-simulation, as well.

\section{Numerical Stability}

Depending on the coupling method, instabilities can still occur for zero-stable coupling methods due to the errors introduced by extra- or interpolation. A weak coupling approach is called numerically stable if it yields a stable solution for a finite macro-step size $H>0$ [9].

To investigate stability properties for finite communication step sizes, we consider a three-mass oscillator as benchmark example, which is illustrated in Figure 3.

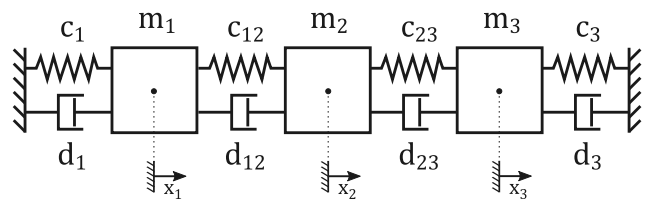

Figure 3: Illustration of a three-mass oscillator. 
The underlying equation system can be interpreted as coupled Dahlquist equations, which can be solved analytically and thus provide an eminently suitable test case. The oscillator with two masses has been taken into consideration in numerous investigations on stability of conventional, single-level co-simulation approaches, where it proves highly sensitive to the choice of parameters and macro step size [9].

For the intended co-simulation, the system is split along the individual masses and coupled via forcedisplacement-coupling (cf. f.i. [10] for further information on the coupling approach), as illustrated in Figure 4.

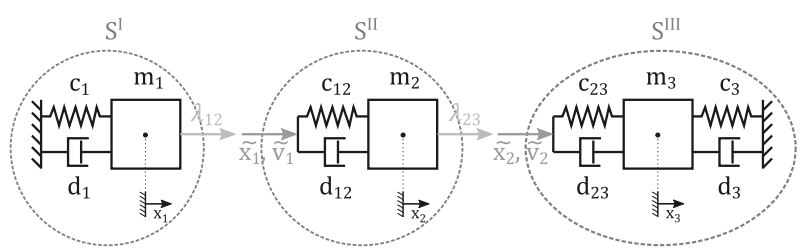

Figure 4: Force-displacement coupling of the three-mass oscillator.

By this coupling approach we obtain the subsystem equations for systems $S^{I}, S^{I I}$ and $S^{I I I}$ :

$$
\begin{aligned}
S^{I}: \quad \dot{x}_{1} & =v_{1} \\
m_{1} \dot{v}_{1} & =-c_{1} x_{1}-d_{1} v_{1}+\lambda_{12} \\
\dot{x}_{2} & =v_{2} \\
S_{2} \dot{v}_{2} & =-c_{12}\left(x_{2}-\widetilde{x_{1}}\right)-d_{12}\left(v_{2}-\widetilde{v_{1}}\right)+\lambda_{23} \\
\dot{x}_{3} & =v_{3} \\
S^{I I I}: \quad m_{3} \dot{v}_{3} & =-c_{23}\left(x_{3}-\widetilde{x_{2}}\right)-d_{23}\left(v_{3}-\widetilde{v_{2}}\right) \\
& +c_{3}\left(-x_{3}\right)+d_{3}\left(-v_{3}\right)
\end{aligned}
$$

With the coupling conditions

$$
\begin{aligned}
\lambda_{12}-c_{12}\left(x_{2}-x_{1}\right)-d_{12}\left(v_{2}-v_{1}\right) & =0 \\
\widetilde{x_{1}}-x_{1} & =0 \\
\widetilde{v_{1}}-v_{1} & =0 \\
\lambda_{23}-c_{23}\left(x_{3}-x_{2}\right)-d_{23}\left(v_{3}-v_{2}\right) & =0 \\
\widetilde{x_{2}}-x_{2} & =0 \\
\widetilde{v_{2}}-v_{2} & =0
\end{aligned}
$$

Following the considerations from section 2, we obtain for matrix $\boldsymbol{D}$ in (3):

$$
\begin{aligned}
\boldsymbol{D} & =\left[\begin{array}{ccccc}
\mathbf{0} & \boldsymbol{D}^{I} \boldsymbol{L}^{I, I I} & \boldsymbol{D}^{I} \boldsymbol{L}^{I, I I I} \\
\boldsymbol{D}^{I I} \boldsymbol{L}^{I I, I} & \mathbf{0} & \boldsymbol{D}^{I I} \boldsymbol{L}^{I I, I I I} \\
\boldsymbol{D}^{I I I} \boldsymbol{L}^{I I I, I} & \boldsymbol{D}^{I I I} \boldsymbol{L}^{I I I, I I} & \mathbf{0}
\end{array}\right] \\
& =\left[\begin{array}{ccccccc}
0 & 0 & 0 & 0 & 0 & 0 \\
0 & 0 & 0 & 0 & 0 & 0 \\
0 & 0 & 0 & 0 & 0 & 0 \\
0 & 0 & 0 & 0 & 0 & 0 \\
-c_{12} & -d_{12} & 0 & 0 & 0 & 0 \\
0 & 0 & -c_{23} & -d_{23} & 0 & 0
\end{array}\right]
\end{aligned}
$$

whence follows $\rho(\boldsymbol{D})=0$, thus guaranteeing zerostability.

For the hierarchical co-simulation approach, systems $S^{I I}$ and $S^{I I I}$ are combined in a second-level co-simulation.

As expected (cf. section 2), we obtain $\rho\left(\boldsymbol{D}_{C S_{1}}\right)=$ $\rho\left(\boldsymbol{D}_{C S_{2}}\right)=0$ for

$$
\boldsymbol{D}_{C S_{1}}=\left[\begin{array}{cccccc}
0 & 0 & 0 & 0 & 0 & 0 \\
0 & 0 & 0 & 0 & 0 & 0 \\
0 & 0 & 0 & 0 & 0 & 0 \\
0 & 0 & 0 & 0 & 0 & 0 \\
-c_{12} & -d_{12} & 0 & 0 & 0 & 0 \\
0 & 0 & 0 & 0 & 0 & 0
\end{array}\right]
$$

and

$$
\boldsymbol{D}_{C S_{2}}=\left[\begin{array}{cccc}
0 & 0 & 0 & 0 \\
0 & 0 & 0 & 0 \\
0 & 0 & 0 & 0 \\
-c_{23} & -d_{23} & 0 & 0
\end{array}\right] \text {, }
$$

thus the conditions for zero-stability are satisfied for both levels of co-simulation.

In the following, several scenarios are performed for both co-simulation approaches to compare numerical stability properties. For all settings, explicit Euler methods are used to solve the individual subsystems. These simple methods have been chosen to enable the focus on the different methods of co-simulation without additional corrections (f.i. by step size control). As synchronization method, Jacobi-type coupling without iteration using zero-order extrapolation for external variables has been used. The initial conditions for all scenarios have been chosen as $x_{1}=1, x_{2}=2, x_{3}=3$ and $v_{1}=v_{2}=v_{3}=0$.

Scenario 1. The parameters for the first scenario to be considered are given in Table 1. 


\begin{tabular}{ccccccccccc}
\hline$c_{1}$ & $c_{12}$ & $c_{23}$ & $c_{3}$ & $d_{1}$ & $d_{12}$ & $d_{23}$ & $d_{3}$ & $m_{1}$ & $m_{2}$ & $m_{3}$ \\
\hline $1 \mathrm{E}-02$ & $1 \mathrm{E}-01$ & 1 & 10 & 0.1 & 0.4 & 1 & 2 & 10 & 10 & 10 \\
\hline
\end{tabular}

Table 1: Parameter settings for Scenario 1.

As can be seen, the spring stiffnesses are chosen to increase from left to right (cf. Figure 3) to result in slower and faster varying subsystems. The step sizes for the individual subsystem solvers are chosen accordingly with $h_{1}=0.005, h_{2}=0.0025, h_{3}=0.00125$. The monolithic reference system is of the form $\dot{\boldsymbol{y}}=\boldsymbol{A} \cdot \boldsymbol{y}$ and can thus be solved analytically. In addition to the analytical solution, the results of the hierarchical co-simulation are compared to a conventional single-level co-simulation. For the latter, a macro step size $H$ of 0.1 seconds is chosen. The results in Figure 5 show that even if the overall communication step size $H_{1}$ is doubled in comparison to the traditional co-simulation, the hierarchical approach yields significantly more accurate results for systems $S^{I I}$ and $S^{I I I}$ if the step size for the second-level co-simulation is chosen adequately $\left(H_{1}=0.2 s, H_{2}=0.05 s\right)$.
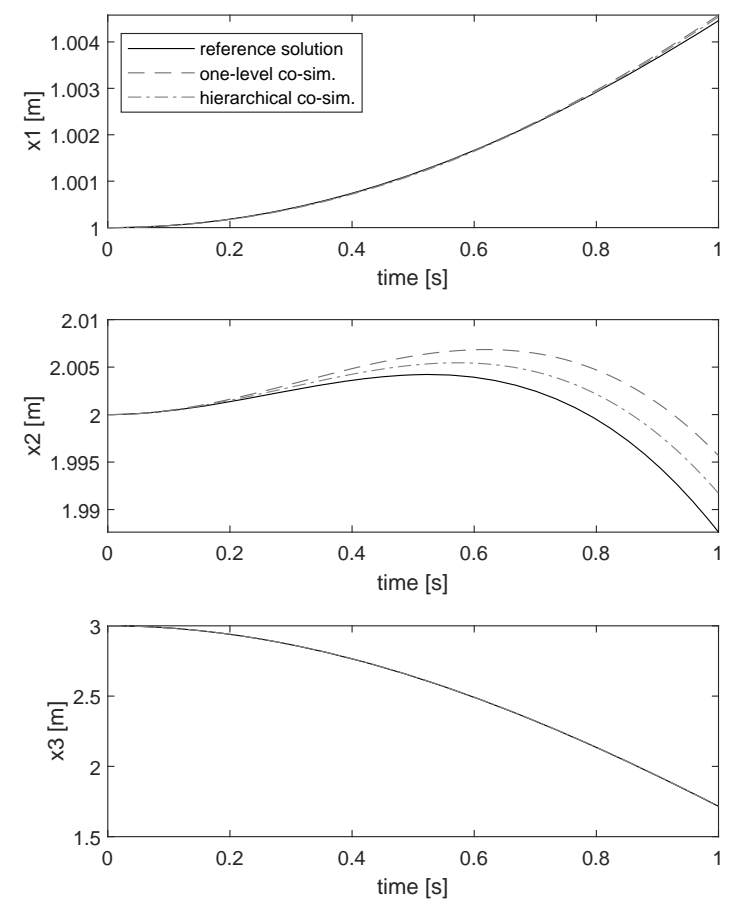

Figure 5: Trajectories of $x_{1}, x_{2}$ and $x_{3}$ for Scenario 1 with $H=0.1 s, H_{1}=0.2 s$ and $H_{2}=0.05 s$.
In spite of plainly distinct errors in specific phases, both approximations remain stable, as can be seen in simulations over a longer period of time.

The maximum absolute errors and elapsed time for several different settings are given in Table 2 .

\begin{tabular}{c|c|cc|cccccc|c}
\hline appr. & $t_{\text {end }}$ & $H \mid H_{1}$ & $H_{2}$ & $\operatorname{err}_{x_{1}}$ & $\operatorname{err}_{v_{1}}$ & $\operatorname{err}_{x_{2}}$ & $\operatorname{err}_{v_{2}}$ & $\operatorname{err}_{x_{3}}$ & $\operatorname{err}_{v_{3}}$ & el. time \\
\hline trad. & 1 & 0.1 & & $1.21 \mathrm{E}-04$ & $5.30 \mathrm{E}-04$ & $8.05 \mathrm{E}-03$ & $1.53 \mathrm{E}-02$ & $9.51 \mathrm{E}-04$ & $6.32 \mathrm{E}-04$ & 0.0066 \\
hier. & 1 & 0.1 & 0.025 & $4.52 \mathrm{E}-05$ & $2.89 \mathrm{E}-04$ & $2.10 \mathrm{E}-03$ & $3.67 \mathrm{E}-03$ & $8.22 \mathrm{E}-04$ & $1.38 \mathrm{E}-03$ & 0.0161 \\
hier. & 1 & 0.2 & 0.05 & $8.26 \mathrm{E}-05$ & $5.34 \mathrm{E}-04$ & $4.08 \mathrm{E}-03$ & $7.44 \mathrm{E}-03$ & $8.51 \mathrm{E}-04$ & $1.06 \mathrm{E}-03$ & 0.0099 \\
trad. & 25 & 0.1 & & $3.96 \mathrm{E}-02$ & $7.13 \mathrm{E}-03$ & $9.35 \mathrm{E}-02$ & $3.68 \mathrm{E}-02$ & $1.78 \mathrm{E}-02$ & $1.24 \mathrm{E}-02$ & 0.1845 \\
hier. & 25 & 0.1 & 0.025 & $1.76 \mathrm{E}-02$ & $3.29 \mathrm{E}-03$ & $2.14 \mathrm{E}-02$ & $8.75 \mathrm{E}-03$ & $6.63 \mathrm{E}-03$ & $5.48 \mathrm{E}-03$ & 0.4018 \\
hier. & 25 & 0.2 & 0.05 & $3.53 \mathrm{E}-02$ & $6.64 \mathrm{E}-03$ & $4.28 \mathrm{E}-02$ & $1.75 \mathrm{E}-02$ & $9.67 \mathrm{E}-03$ & $7.20 \mathrm{E}-03$ & 0.2514 \\
\hline
\end{tabular}

Table 2: Maximum error and elapsed time for the traditional and hierarchical co-simulation approach in Scenario 1 .

We see that while the execution time is significantly higher in case of the same step size on the upper level and the traditional co-simulation - which has to be expected due to the additional synchronization on the lower level the high difference can be overcome while still maintaining better accuracy by increasing both macro step sizes in the hierarchical approach.

Scenario 2. In Scenario 2, the stiffnesses differ to a greater extent (see parameters in Table 3), which can lead to stability issues if communication step sizes are chosen too large. The conventional co-simulation already yields unstable results for the same step size as in Scenario 1 $(H=0.1)$. The solution obtained by the hierarchical approach with the same upper-level communication step size but additional synchronization between subsystems $S^{I I}$ and $S^{I I}$ remains stable.

\begin{tabular}{ccccccccccc}
\hline$c_{1}$ & $c_{12}$ & $c_{23}$ & $c_{3}$ & $d_{1}$ & $d_{12}$ & $d_{23}$ & $d_{3}$ & $m_{1}$ & $m_{2}$ & $m_{3}$ \\
\hline $1 \mathrm{E}-03$ & $1 \mathrm{E}-01$ & 10 & 100 & 0.1 & 0.4 & 1 & 2 & 10 & 10 & 10 \\
\hline
\end{tabular}

Table 3: Parameter settings for Scenario 2.

Even for a larger communication step size on the upper level $\left(H_{1}=0.2\right)$, stability is maintained with the hierarchical approach, as the coupling between systems $S^{I I}$ and $S^{I I}$ is the crucial one (cf. Figure 6).

If the synchronization time on the second level is also increased (to $H_{2}=0.05$ ), qualitative behavior is still maintained but errors are too high to consider the solution still acceptable (cf. Table 4). 

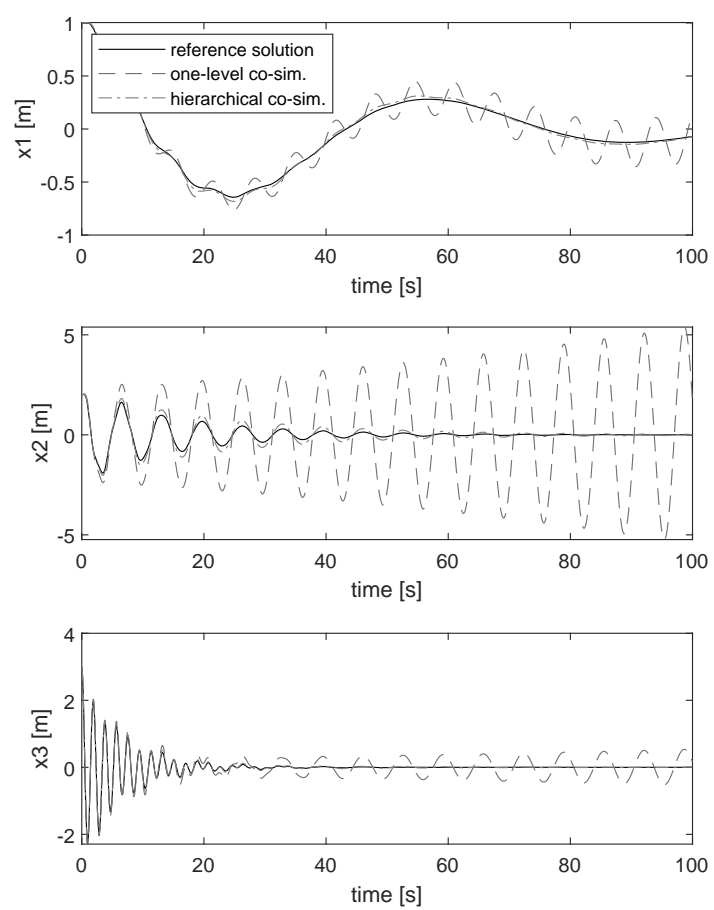

Figure 6: Trajectories of $x_{1}, x_{2}$ and $x_{3}$ for Scenario 2 with $H=0.1 s, H_{1}=0.2 s$ and $H_{2}=0.025 s$ from $t_{\text {start }}=0 s$ to $t_{\text {end }}=100 s$.

Scenario 3. In Scenario 3, the stiffnesses for the springs attached to mass $m_{1}$ are increased, too (see Table 5), which leads to unstable results for the traditional as well as hierarchical approach with step sizes $H=0.1 s$, $H_{1}=0.1 s$ and $H_{2}=0.025 s$, see Figure 7. This makes sense as the increased stiffness for System $I$ can not be compensated by closer communication of Systems $I I$ and III.

The macro step sizes $H$ and $H_{1}$ would have to be chosen as low as 0.03 to keep the error in bounds at all, even though results are still too far from the reference solution to be of use.

\section{Conclusion}

In this paper, the method of hierarchical co-simulation has been presented and investigated with respect to stability properties. In comparison to hierarchical multirate approaches as presented in [5, 12], the application of the hierarchical co-simulation method presented in this paper

\begin{tabular}{c|c|cc|cccccc|c}
\hline appr. & $t_{\text {end }}$ & $H \mid H_{1}$ & $H_{2}$ & err $_{x_{1}}$ & $\operatorname{err}_{v_{1}}$ & err $_{X_{2}}$ & $\operatorname{err}_{v_{2}}$ & $\operatorname{err}_{X_{3}}$ & $e r r_{v_{3}}$ & el. time \\
\hline trad. & 3 & 0.1 & & $1.19 \mathrm{E}-02$ & $1.56 \mathrm{E}-02$ & $4.03 \mathrm{E}-01$ & $4.61 \mathrm{E}-01$ & $9.78 \mathrm{E}-02$ & $2.37 \mathrm{E}-01$ & 0.0202 \\
hier. & 3 & 0.1 & 0.025 & $7.59 \mathrm{E}-03$ & $7.01 \mathrm{E}-03$ & $9.54 \mathrm{E}-02$ & $1.10 \mathrm{E}-01$ & $5.01 \mathrm{E}-02$ & $1.33 \mathrm{E}-01$ & 0.0376 \\
hier. & 3 & 0.2 & 0.025 & $1.54 \mathrm{E}-02$ & $1.44 \mathrm{E}-02$ & $1.94 \mathrm{E}-01$ & $2.24 \mathrm{E}-01$ & $6.56 \mathrm{E}-02$ & $1.66 \mathrm{E}-01$ & 0.0372 \\
hier. & 3 & 0.2 & 0.05 & $1.46 \mathrm{E}-02$ & $1.29 \mathrm{E}-02$ & $9.48 \mathrm{E}-02$ & $1.10 \mathrm{E}-01$ & $5.00 \mathrm{E}-02$ & $1.33 \mathrm{E}-01$ & 0.0258 \\
trad. & 100 & 0.1 & & $2.37 \mathrm{E}-01$ & $2.28 \mathrm{E}-01$ & $5.38 \mathrm{E}+00$ & $5.06 \mathrm{E}+00$ & $5.35 \mathrm{E}-01$ & $5.06 \mathrm{E}-01$ & 2.2885 \\
hier. & 100 & 0.1 & 0.025 & $2.57 \mathrm{E}-02$ & $1.30 \mathrm{E}-02$ & $2.90 \mathrm{E}-01$ & $2.79 \mathrm{E}-01$ & $6.86 \mathrm{E}-02$ & $1.98 \mathrm{E}-01$ & 6.4143 \\
hier. & 100 & 0.2 & 0.025 & $5.81 \mathrm{E}-02$ & $3.08 \mathrm{E}-02$ & $6.96 \mathrm{E}-01$ & $6.64 \mathrm{E}-01$ & $1.06 \mathrm{E}-01$ & $2.53 \mathrm{E}-01$ & 5.3847 \\
hier. & 100 & 0.2 & 0.05 & $3.90 \mathrm{E}-02$ & $1.47 \mathrm{E}-02$ & $2.88 \mathrm{E}-01$ & $2.77 \mathrm{E}-01$ & $6.85 \mathrm{E}-02$ & $1.98 \mathrm{E}-01$ & 2.5811 \\
\hline
\end{tabular}

Table 4: Maximum error and elapsed time for the traditional and hierarchical co-simulation approach in Scenario 2.

\begin{tabular}{ccccccccccc}
\hline$c_{1}$ & $c_{12}$ & $c_{23}$ & $c_{3}$ & $d_{1}$ & $d_{12}$ & $d_{23}$ & $d_{3}$ & $m_{1}$ & $m_{2}$ & $m_{3}$ \\
\hline 1 & 10 & 10 & 100 & 0.1 & 0.4 & 1 & 2 & 10 & 10 & 10 \\
\hline
\end{tabular}

Table 5: Parameter settings for Scenario 3.
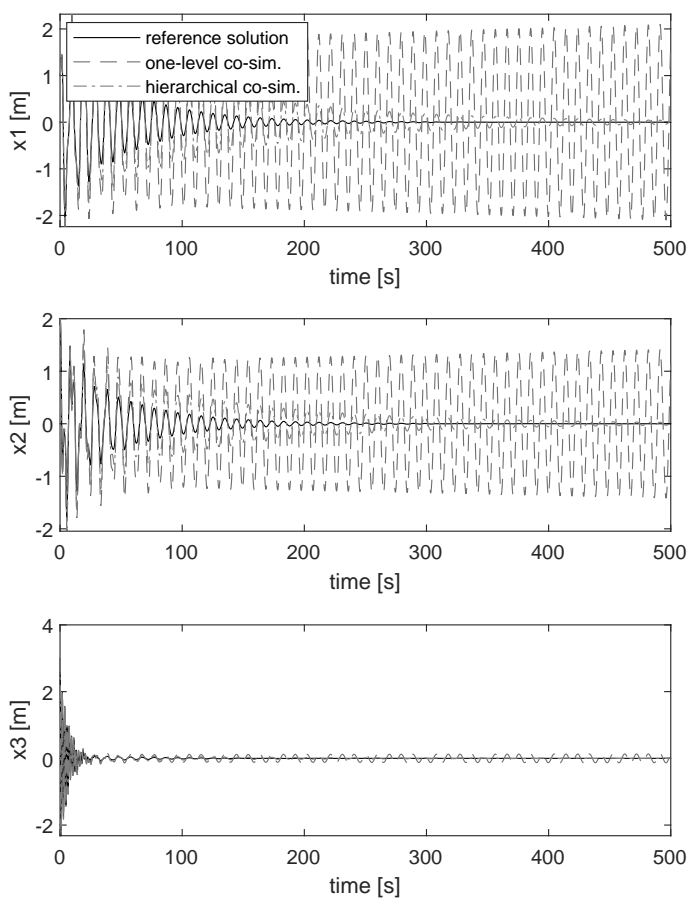

Figure 7: Trajectories of $x_{1}, x_{2}$ and $x_{3}$ for Scenario 3 with $H=0.1 s, H_{1}=0.1 s$ and $H_{2}=0.025 s$ from $t_{\text {start }}=0 s$ to $t_{\text {end }}=100$ s.

does not require any knowledge on the underlying system per se. The subsystems can, as in common co-simulation methods, be treated as black boxes with information on the input and output dependencies without interfering 
with the subsystem solvers. This can be beneficial when using co-simulation platforms like the BCVTB [11] or standards like the $\mathrm{FMI}^{1}$, and in particular for interdisciplinary collaborative projects where partial systems are developed independently and possibly protected by company-specific privacy agreements.

While in the experiments above, quite simple subsystem solvers and coupling methods are chosen, improvement methods commonly used in single-level co-simulation approaches like variations of extrapolation order, coupling methods (sequential or mixed algorithms and waveform iteration) and stabilization techniques can of course be utilized in hierarchical co-simulation as well. Detailed studies on the advantages of said techniques for traditional co-simulation are ample in the literature (see for example $[13,14,15,16])$. In addition, the results from section 3 show that stability issues can be tackled by introducing another layer of communication instead of having to decrease the overall communication step size, thus providing an innovative method for stabilization.

\section{References}

[1] Hafner I, Niki P. On the Terminology and Structuring of Co-simulation Methods. In Proceedings of the 8th International Workshop on Equation-Based Object-Oriented Modeling Languages and Tools. EOOLT '17; 2017; Weßling, Germany. New York, USA: ACM. 67-76. doi: 10.1145/3158191.3158203.

[2] Zeigler BP. Object-Oriented Simulation with Hierarchical, Modular Models: Intelligent Agents and Endomorphic Systems.. Saint Louis: Elsevier Science; 2014.

[3] Morvan G. Multi-level agent-based modeling - A literature survey. arXiv:1205.0561 [cs]. 2013.

[4] Scattolini R. Architectures for distributed and hierarchical Model Predictive Control - A review. Journal of Process Control. 2009; 19(5): 723-731. doi: 10.1016/j.jprocont.2009.02.003.

[5] Günther M, Rentrop P. Partitioning and Multirate Strategies in Latent Electric Circuits. In: Bank RE, Gajewski H, Bulirsch R, Merten K. Mathematical Modelling and Simulation of Electrical Circuits and Semiconductor Devices. Basel: Birkhäuser; 1994. p 33-60. doi: 10.1007/978-3-0348-8528-7_3

[6] Trcka, M. Cosimulation for Performance Prediction of Innovative Integrated Mechanical Energy Systems in

\footnotetext{
${ }^{1}$ https://fmi-standard.org/
}

Buildings [dissertation]. Technische Universiteit Eindhoven; 2008.

[7] Kübler R, Schiehlen W. Two Methods of Simulator Coupling. Mathematical and Computer Modelling of Dynamical Systems. 2000; 6(2): 93-113. doi: 10.1076/1387-3954(200006)6:2;1-M;FT093.

[8] Horn RA, Johnson CR. Matrix analysis. 23. print. Cambridge: Cambridge Univ. Press; 2010.

[9] Busch, M. Zur effizienten Kopplung von Simulationsprogrammen. Kassel: Kassel University Press; 2012.

[10] Schweizer B, Lu D. Semi-implicit co-simulation approach for solver coupling. Archive of Applied Mechanics. 2014; 84(12): 1739-1769. doi: 10.1007/s00419-014-0883-5.

[11] Wetter M. Co-simulation of building energy and control systems with the Building Controls Virtual Test Bed. Journal of Building Performance Simulation. 2011; 4(3): 185-203. doi: 10.1080/19401493.2010.518631.

[12] Striebel, M. Hierarchical Mixed Multirating for Distributed Integration of DAE Network Equations in Chip Design [dissertation]. Bergische Universität Wuppertal; 2006.

[13] White J, Odeh F, Sangiovanni-Vincentelli AL, Ruehli AE. Waveform Relaxation: Theory and Practice [Technical Report]. [EECS Department]. University of California; 1985.

[14] Larsson J, Krus P. Stability Analysis of Coupled Simulation. In Dynamic Systems and Control, Volumes 1 and 2. ASME International Mechanical Engineering Congress and Exposition. 2003 Nov; Washington DC, USA. 861-868. doi: 10.1115/IMECE2003-41192.

[15] Arnold M. Stability of Sequential Modular Time Integration Methods for Coupled Multibody System Models. Journal of Computational and Nonlinear Dynamics. 2010; 5(3): 031003. doi: 10.1115/1.4001389.

[16] Schweizer B, Li P, Lu D, Meyer T. Stabilized implicit co-simulation methods: solver coupling based on constitutive laws. Archive of Applied Mechanics. 2015; 85(11): 1559-1594. doi: 10.1007/s00419-015-0999-2.

[17] Arnold M, Clauss C, Schierz T. Error Analysis and Error Estimates for Co-Simulation in FMI for Model Exchange and Co-Simulation V2.0. Archive of Mechanical Engineering. 2013; LX(1). doi: 10.2478/meceng2013-0005. 